\title{
Evaluación de la efectividad de la toxina botulínica en la disfonía espasmódica aductora mediante el análisis acústico
}

\author{
LAURA RIERA TUR \\ Hospital Universitario Puerta del \\ Mar \\ Otorrinolaringología \\ Avda. Ana de Viya 21 \\ 11009 Cádiz \\ E-mail: laurarieratur@gmail.com
}

Dolores Alonso Blanco

Hospital Universitario Puerta del

Mar

Otorrinolaringología

Avda. Ana de Viya 21

11009 Cádiz

E-mail: lola.ab87@gmail.com

EVALUACIÓN DE LA EFECTIVIDAD DE LA TOXINA BOTULÍNICA EN LA DISFONÍA ESPASMÓDICA ADUCTORA MEDIANTE EL ANÁLISIS ACÚSTICO

RESUMEN: Introducción: La disfonía espasmódica es una distonía focal caracterizada por contracciones involuntarias disritmicas de la musculatura de la cuerda vocal, resultando una voz con contracciones espasmódicas que alteran el ritmo del habla y la capacidad de comunicación del paciente. Métodos: Presentamos una serie de 113 pacientes con diagnóstico de disfonía espasmódica aductora con edades comprendidas entre los 18 y 65 años. Realizaremos a todos ellos un análisis acústico de la voz antes y después del tratamiento con toxina botulínica. Resultados: Observamos una normalización de todos los parámetros en el análisis acústico tras el tratamiento con toxina botulínica. Conclusión: La infiltración de toxina botulinica en las cuerdas vocales es un tratamiento efectivo en la disfonia espasmódica aductora y el análisis acústico de la voz es un buen método para verificarlo.

PALABRAS CLAVES: distonía laringea; trastornos de la voz; disfonía espasmódica; análisis acústico; toxina botulinica.

SUMARIO: 1. Introducción. 1.1. Forma de inicio. 1.2. Signos subjetivos. 1.3. Signos objetivos. 1.4. Diagnóstico. 1.5. Tratamiento. 1.6. Evolución sin tratamiento adecuado. 1.7. Objetivos. 2. Materiales y métodos. 3. Resultados. 4. Discusión. 5. Conclusiones.

\author{
Miguel De Mier MoRAles \\ Hospital Universitario Puerta del \\ Mar \\ Otorrinolaringología \\ Avda. Ana de Viya 21 \\ 11009 Cádiz \\ E-mail: demiermiguel@gmail.com
}

Gumersindo Jesus Pérez Ortega

Hospital Universitario Puerta del Mar

Otorrinolaringología

Avda. Ana de Viya 21

11009 Cádiz

E-mail: gumersindoje-

sus@gmail.com

EVALUATION OF THE EFFECTIVENESS OF BOTULINUM TOXIN IN ADDUCTOR SPASMODIC DYSPHONIA THROUGH ACOUSTIC ANALYSIS

ABSTRACT: Introduction: Spasmodic dysphonia is a focal dystonia characterized by involuntary dysrhythmic contractions of the musculature of the vocal cord, resulting in a voice with spasmodic contractions that alter the speech rhythm and the patient's communication capacity. Methods: We present a series of 113 patients di agnosed with adductor spasmodic dysphonia with ages between 18 and 65 years. We will perform an acoustic analysis of the voice before and after the treatment with botulinum toxin. Results: We observed a normalization of all parameters in the acoustic analysis after treatment with botulinum toxin. Conclusion: The infiltration of botulinum toxin in the vocal cords is an effective treatment in adductor spasmodic dysphonia and the acoustic analysis of the voice is a good method to verify it.

KEY WORDS: laryngeal dystonia; voice disorders; spasmodic dysphonia; acoustic analysis; botulinum toxin.

SUMMARY: 1. Introduction. 1.1 Start form. 1.2. Subjective signs. 1.3. Objective signs. 1.4. Diagnosis. 1.5. Treatment. 1.6. Evolution without adequate treatment. 1.7. Objectives. 2. Materials and methods. 3. Results. 4. Discussion. 5. Conclusions.
Encarnación Ávalos Serrano

Hospital Universitario Puerta del

Mar

Otorrinolaringología

Avda. Ana de Viya 21

11009 Cádiz

E-mail: e.avalos@comcadiz.es

Juan Manuel Montesinos GonzÁlez

Hospital Universitario Puerta del

Mar

Otorrinolaringología

Avda. Ana de Viya 21

11009 Cádiz

E-mail: montego_rota@comcadiz.es

ÉVALUATION DE L'EFFICACITE DE LA TOXINE BOTULIQUE DANS LA DYSPHONIE SPASMODIQUE ADDUCTEUR PAR ANALYSE ACOUSTIQUE

RÉSUMÉ: Introducción: La dysphonie spasmodique est une dystonie focale caractérisée par des contractions involontaires dysrythmiques de la musculature de la corde vocale entraînant une voix avec des contractions spasmodiques qui modifient le rythme de la parole et la capacité de communication du patient. Méthodes : Nous présentons une serie de 113 patients compris entre 18 et 65 ans pour lesquels nous réaliserons une analyse acoustique de la voix avant et après le traitement par toxine. Résultats : Suite au traitement par la toxine botulique de type A, nous observons une normalisation de l'ensemble des paramètres de l'analyse acoustique. Discussion/conclusion :

L'infiltration de la toxine botulique dans les cordes vocales est un traitement efficace pour la dysphonie spasmodique en adduction qui permet d'obtenir une amélioration des paramètres

MOTS CLÉS: dystonie laryngée; troubles de la voix (parole); dysphonie spasmodique; analyse acoustique; toxine botulique.

SOMMAIRE: 1 . Introduction. 1.1. Formulaire de départ. 1.2. Signes subjectifs. 1.3. Signes objectifs. 1.4. Diagnostic. 1.5.Traitement. 1.6 . Evolution sans traitement adéquat. 1.7. Objectifs. 2. Matériels et méthodes. 3.Résultats. 4. Discussion. 5. Conclusions.
Fecha de Recepción Fecha de Revisión Fecha de Aceptación Fecha de Publicación
$05 / 02 / 2019$

$07 / 03 / 2019$

$10 / 05 / 2019$

$01 / 12 / 2020$ 


\section{Evaluación de la efectividad de la toxina botulínica en la disfonía espasmódica aductora mediante el análisis acústico}

Laura Riera Tur, Miguel de Mier Morales, Encarnación Ávalos Serrano, Dolores Alonso Blanco, Gumersindo Jesus Pérez Ortega \& Juan Manuel Montesinos GonZÁlez

\section{INTRODUCCIÓN}

La disfonía espasmódica es un trastorno de la voz originado por una interrupción o disrupción del control motor laríngeo, dando lugar a movimientos involuntarios de la musculatura laringea durante la fonación.

La disfonía espasmódica es un tipo de distonía, otros ejemplos serian la distonía cervical, el blefaroespasmo, distonía del escribiente etc. Se trata de una condición crónica secundaria a una alteración neurológica de la motoneurona central que origina espasmos musculares focales.

Los primeros casos de disfonía espasmódica se describieron por Traube y por Shnitzler con la denominación de disfonía espástica. Inicialmente se creía que la etiología de la enfermedad era psicológica, sin embargo, a partir de los trabajos de Cannito (1981) y Whurr (1993), conocemos la naturaleza neurológica de esta enfermedad aunque los mecanismos neurofisiopatológicos todavía permanecen poco claros.

La disfonía espasmódica en España se considera una enfermedad rara. La prevalencia estimada se encuentra en 5.9 por 100.000. Sin embargo, se cree que se trata de una patología infradiagnosticada con más de 5.000 casos sin diagnosticar. Es más frecuente en mujeres que en varones con una proporción de 3 a 1 y existe un pico de incidencia entre los 35 y los 45 años.

Dentro de la disfonía espasmódica, diferenciamos tres grupos:

- Disfonía espasmódica aductora: Se produce una hiperaducción irregular de las cuerdas vocales con excesivo cierre glótico. Es el subtipo más frecuente, suponiendo el 90\% de los casos diagnosticados.

Los pacientes se caracterizan por presentar una voz ahogada, estrangulada, constreñida, tensa y con saltos abruptos en la fonación en medio de las vocales.

Las roturas son debidas al cierre brusco de la glotis por hiperaducción que interrumpe el flujo de aire a través de las cuerdas vocales.

- Disfonía espasmódica abductora en la que se produce una aproximación incompleta e irregular de las cuerdas vocales. Supone el 10\% de los pacientes con disfonía espasmódica.

En este caso se trata de una voz aérea, con gran esfuerzo, entrecortada y con rupturas bruscas que origina susurros durante la fonación. Existe una excesiva y prolongada abducción en consonantes sordas (/h/,/s/,/f/,/p/,/t/,/k/). En casos graves se puede producir la afonía completa.

- Forma mixta en la que ambos elementos están presentes. 


\subsection{FORMA DE INICIO}

Por lo general el inicio de los trastornos es progresivo e insidioso.

El paciente presenta al principio crisis aisladas que afectan a algunas sílabas. Durante algunos segundos parece que su voz "se estrangula en la garganta", con sensación de astricción. Sin embargo, estas crisis son durante mucho tiempo apenas perceptibles para el interlocutor, aunque muy molestas e inquietantes para el paciente debido a su carácter imprevisible y desconcertante.

Poco a poco, por etapas, las crisis se hacen más frecuentes y audibles. En ocasiones la voz presenta bruscos tránsitos a falsete. Al mismo tiempo, la tensión del paciente se hace cada vez más evidente, y este transmite la impresión de estar sometido a un control permanente.

El aspecto más o menos congestivo de los repliegues vocales, consecutivo al esfuerzo vocal, da pie con frecuencia a la prescripción de un tratamiento antinflamatorio o antiinfeccioso, que es por completo ineficaz. Se recomienda a veces una cura de silencio vocal cuyo efecto suele ser agravante: al finalizar la cura (10 días, a veces más) el paciente comprueba con bastante frecuencia que sus dificultades como minimo se han duplicado.

Por otra parte, a veces el interrogatorio detecta la existencia de un periodo de importantes dificultades por el que acaba de pasar el paciente, de causa familiar (luto) o profesional. La disfonía suele aparecer cuando el paciente comienza a superar su problema.

\subsection{SigNOS SUBJETIVOS}

Una vez que aparece el trastorno, el paciente suele experimentar sensaciones de opresión respiratoria y falta de soplo. Otras veces refiere sensaciones de astricción y bloqueo en el cuello y, con menos frecuencia, contracturas abdominales y dolor al deglutir y articular.

En lo que respecta a la voz, lo que produce más desazón es su carácter imprevisible. La voz normal se bloquea a veces y otras exige un esfuerzo intenso. En algunos momentos, a pesar de este, el paciente tiene la impresión de que no va a llegar a finalizar la frase que ha iniciado.

Por lo común, el paciente subraya que su voz es mejor por la mañana y cuando está tranquilo, alterándose con la fatiga y el nerviosismo; sin embargo, en muchos casos comprueba que su voz mejora a medida que va hablando.

Dada la intensidad del esfuerzo que debe realizar para emitir sonidos, algunos pacientes hablan de "estreñimiento verbal". Otros comentan que mientras rien su voz se normaliza por completo (es el signo de la risa). La ingesta de alcohol y la sorpresa producen a menudo el mismo resultado. 


\subsection{SigNOS OBJETIVOS}

Debemos destacar que no siempre es oportuno insistir en la evidencia de signos fónicos objetivos. En especial es conveniente acortar las pruebas de lectura, de voz de llamada y de voz cantada una vez aparecidos los primeros espasmos: el comportamiento de esfuerzo intenso realizado por el paciente puede ser traumático y agravar su problema. Asimismo, hay que evitar enfrentarle con excesiva brusquedad con la amplitud del deterioro de su función vocal.

En este proceso los espasmos respiratorios y vocales son los que verdaderamente caracterizan la emisión vocal, facilitando el diagnóstico simplemente al oír la voz.

En su forma típica los espasmos respiratorios son el resultado de una intensa tensión respiratoria que implica los músculos respiratorios (diafragma, intercostales, músculos cervicales) y espiratorios (torácicos y abdominales), y que provoca una emisión vocal entrecortada, vibrante, con una especie de sacudidas que causan irregularidades en la intensidad de la voz. A veces un temblor regular se superpone a esta irregularidad del débito. La voz se presenta globalmente tensa, contenida e irregular, además, está agravada y es poco intensa y a veces nasal. En algunos casos se observan emisiones vocales invertidas, produciéndose a ratos la voz en la inspiración (voz invertida).

En otros casos, los espasmos espiratorios desencadenan la desodorización completa de algunas sílabas, algunas palabras o, incluso, algunos finales de frase. En ocasiones también puede observarse una evolución a una afonia parcial o completa.

Cuando los espasmos respiratorios son dominantes, se habla clásicamente de disfonía espasmódica en abducción, con la salvedad de que en este caso se trata de una distonía de los músculos cricoaritenoideos posteriores. Es muy posible que en este caso pudiésemos observar un trazado de hiperinervación patológica en los músculos que clásicamente son considerados como los únicos abductores de los repliegues vocales, no sin señalar que su disposición anatómica y sus inserciones explican mal por qué el borde libre de los repliegues vocales se mantiene rectilineo (teoría de Ganz) durante la apertura activa de la glotis. Pero en todo caso, en las disfonías espasmódicas, denominadas en abducción, lo más esencial de la distonía afecta ciertamente más a la musculatura respiratoria que a la musculatura laríngea. Esto explica sin duda que, como desarrollamos más adelante, la infiltración de toxina botulínica de los músculos supuestamente responsables sea menos eficaz en esta forma clinica.

En cuanto a la voz conversacional, es importante subrayar que a un oyente no experimentado puede parecerle completamente normal, a pesar de la importante dificultad que experimenta el paciente. Un oído más experimentado notará, sin embargo, la existencia de ciertas irregularidades en la 
intensidad de determinadas vocales, algunos aflojamientos del soplo o una caracteristica actitud de control del habla.

Se suele apreciar que el trastorno varía de un momento a otro y que se agrava cuando el paciente presta atención a su voz o está emocionado. Por el contrario, al reirse, el trastorno desaparece por completo: es el signo de la risa de Garde. La explicación es bastante sencilla: según Spencer (1908), la risa corresponde a una descarga de energia que reduce repentinamente la "tensión interior". Asimismo, la alteración desaparece después de bostezar, lo que se explica de la misma manera. También puede desaparecer totalmente, aunque de forma provisional, cuando el paciente produce una voz inhabitual, por ejemplo, una voz canturreante, una voz de falsete o una voz más potente.

Por lo general, la lectura, sobre todo la proyectada, agrava de manera considerable las dificultades de la emisión vocal. En esta ocasión a veces se observa un bloqueo completo de la voz. Sin embargo, en otros casos, la lectura facilita la emisión vocal.

Habitualmente la llamada es imposible con voz de pecho: los diversos intentos culminan en un bloqueo absoluto. Por el contrario, el "yu-ju" femenino con voz "de cabeza" se efectúa a veces a la perfección. Debemos recordar que la alteración de llamada constituye en ocasiones el primer signo del trastorno. No obstante, paradójicamente esta voz de llamada puede estar relativamente conservada pese a que esté muy alterada la voz conversacional.

La voz cantada es a menudo imposible o está muy desorganizada. En los demás casos, puede estar alterada completamente o en parte, unas veces en los agudos y otras en los graves.

\subsection{Diagnóstico}

Para el diagnóstico será necesario realizar una anamnesis exhaustiva complementada con una buena exploración física. Todo diagnóstico debe ir procedido de una valoración por Neurología, para descartar posibles patologías asociadas u otros diagnósticos.

Los pacientes suelen referir una clínica de empeoramiento de la calidad de la voz en situaciones de estrés o cuando hablan por teléfono. Habitualmente se produce una mejoría parcial de la voz con la toma de sedantes como el alcohol y las benzodiacepinas y, además, en las situaciones en la que el paciente canta o rie, la voz se desarrolla con mayor fluidez.

En la exploración física incluiremos una exploración neurológica básica y una exploración otorrinolaringológica orientada a la valoración de la voz que incluya:

- Valoración psicoacústica (GRABS).

- Exploración laringoscópica.

- Videolaringoestroboscopia. 
- Análisis acústico: Jitter y Shimmer. Espectrograma y frecuencia fundamental

En ocasiones será necesario solicitar pruebas de imagen complementarias como la RMN o el TC.

El diagnóstico diferencial principalmente lo realizaremos con la Esclerosis Lateral Amiotrófica, la disfonía de conversión, la disfonía hipertónica, la voz de los parkinsonianos y el temblor esencial.

\subsection{Tratamiento}

La reeducación vocal en la disfonía espasmódica es relativamente ineficaz (Boone, 2014; Vogel, 2001). Cuando se combina con terapia farmacológica puede mejorar la calidad de la voz y prolongar la ventaja de los efectos farmacológicos (Stemple, 2000; Murry, 1995). Se pueden esperar resultados interesantes cuando esta reeducación se inicia por un entrenamiento en la relajación y en ejercicios orientados hacia la reconstrucción del esquema corporal y en el dominio del soplo, antes de abordar (con prudencia y sin insistir) algunos ensayos de producción vocal controlada (Stemple, 2000; Vogel, 2001; Murry 1995).

Si bien antes de esta "era botulínica" la impresión generalmente dominante era la de la incurabilidad de esta afección, los restantes procedimientos no están exentos de eficacia y algunos siguen de actualidad como terapia coadyuvante o como tratamiento de soporte.

\section{- Tratamiento antinflamatorio}

A falta de otro mejor, este tratamiento se ha prescrito ya que, en efecto, la mucosa laringea puede estar irritada por el sobreesfuerzo vocal derivado de la disfonía. Se comprende fácilmente que no se pueda obtener una eficacia real, puesto que carece de acción sobre el proceso distónico existente.

\section{- Silencio vocal y reposo}

Prescrito a veces durante un periodo de varios dias, hasta 2-3 semanas, este tratamiento se revela no solo ineficaz, sino también nocivo. Se aprecia con regularidad en las observaciones del paciente, que, confiando en este tratamiento estricto que se ha seguido escrupulosamente, constata que al final de este periodo de silencio sus dificultades se han amplificado, como si la detención del funcionamiento de su mecánica vocal hubiese aumentado un punto la tendencia de ésta al bloquearse. La explicación de este efecto nocivo del reposo vocal en la disfonía espasmódica no está clara. Esto no impide que se trate de una contraindicación absoluta en esta afección. E1 reposo general, que a veces se aconseja en los casos en los que el paciente 
presenta la tendencia al ejercicio física intenso que se ha señalado anteriormente, se manifiesta igual de catastrófico, tanto para el equilibrio del paciente como para su función vocal.

\section{- Tratamiento farmacológico}

No existe ningún tratamiento farmacológico que, administrado por vía general, resulte curativo para la disfonía espasmódica. Se han ensayado muchos productos, pero sus efectos son transitorios y su interés se ve reducido por la aparición de fenómenos de habituación. Citaremos, sin embargo, el diazepam, el cloracepato dipotásico y a la codeína, que, a la espera de un tratamiento más eficaz, puedan atenuar las dificultades en determinadas circunstancias en las que el paciente necesita mejorar la voz.

\section{- Reeducación vocal}

En la actualidad, de ningún modo debemos plantearnos la reeducación vocal aisladamente. Pensamos que debe llevarse a cabo siempre que sea posible, junto con la infiltración botulínica de los repliegues vocales. En efecto, dado que los espasmos vocales constituyen una molestia notable para el paciente, al parecer es urgente hacerlos desaparecer sin demora, de forma que marcamos una interrupción del proceso distónico que se alimenta de los esfuerzos ciegos realizados por el paciente para hablar a pesar de los espasmos. La botulinización crea una pausa en esta lucha patógena, que permite que la reeducación actúe de forma infinitamente más eficaz que en el pasado.

Esta reeducación pasa por la construcción del esquema corporal. Para ello, como en el caso de disfonías disfuncionales habituales, se comienza con ejercicios en decúbito. No lo haremos con la relajación con los ojos abiertos, a menudo inaccesible para este tipo de pacientes, sino más bien por la respiración del remador, para pasar inmediatamente al soplo rítmico y después eventualmente al ejercicio del hara.

Respecto a los ejercicios vocales, durante mucho tiempo, o puede que incluso hasta el final de la reeducación, se limitarán a algunas producciones sonoras al final de las sesiones, lo justo para hacer sentir al paciente que no hemos perdido de vista su problema vocal.

\section{- Psicoterapia}

Ciertamente, la adopción de este tratamiento parece ser bastante lógica, en la medida en que está fuera de duda que en esta afección existen factores psicológicos, ya sea en tanto que factores favorecedores o en tanto que consecuencia de las dificultades en la vida de relación causadas por la alteración vocal. Sin embargo, hay que admitir que cuando se ha desencadenado el proceso distónico, la psicoterapia aislada se torna ineficaz, tanto más 
cuanto el paciente no ha solicitado dicho tratamiento. Esta, sin embargo, puede producirse como continuación del tratamiento reeducativo. En este caso, la indicación principal es la expresión escénica.

\section{- Tratamiento quirúrgico}

En 1975, en San Francisco, Dedo propuso tratar la disfonía espasmódica mediante la sección del nervio recurrente, con preferencia el izquierdo porque, al ser más largo, está más expuesto que el derecho a cualquier lesión. Esta sección de un nervio recurrente se traduce en la inmovilidad de un repliegue vocal y en la desaparición inmediata y muy espectacular de los espasmos vocales.

Incluso si esta intervención ha marcado un hito en la historia del tratamiento de la disfonía espasmódica, los resultados a largo plazo son, pese a todo, bastante decepcionantes, teniendo en cuenta la mutilación definitiva que causa la intervención.

En el año 80 Isshiki et al. introdujeron la cirugía del escudo tiroideo (tiroplastia) en pacientes con disfonía espasmódica. Esta técnica permite ajustar la posición y la tensión de los pliegues vocales.

En 1982, Frèche propuso una intervención menos destructiva que consiste en electrocoagular en un repliegue vocal las terminaciones nerviosas del recurrente. Esta determina, por fuera de la apófisis vocal, un cráter de $8 \mathrm{~mm}$ de diámetro por $5 \mathrm{~mm}$ de profundidad. Como en la intervención de Dedo, es seguida por una inmovilidad del repliegue vocal operado y una desaparición de los espasmos vocales.

A pesar de que esta intervención supone un gran avance frente a la de Dedo, hoy en día está impuesta la infiltración botulínica. Blitzer et al. aplicaron la inyección de toxina botulínica en 1984.

\section{- Inyecciones localizadas de toxina botulínica}

La toxina botulinica, producida por la bacteria Clostridium botulinum, es una potente neurotoxina que inhibe la liberación de acetilcolina en el extremo terminal de las motoneuronas a la altura de la unión neuromuscular, inhibiendo así la transmisión del impulso nerviosos, dando lugar a una paresia o una parálisis temporal del músculo inyectado (Blitzer et al., 2001). Se han publicado una gran cantidad de artículos que han descrito el uso de la toxina botulínica en el tratamiento de la disfonía espasmódica.

Las características de la intervención realizada y las variables de las medidas de resultados difieren de unos estudios a otros. La respuesta a la toxina botulinica ha sido valorada mediante cambios en la electroglotografia, electromiografia, estudio acústico, fibroendoscopia, estroboscopia, tiempo de efecto del tratamiento o estudio aerodinámico, así como cambios en la calidad de vida relacionada con la voz (Esposito, 2015; Singh, 2016; Rojas, 2017; Faham, 2019). Con respecto a la técnica empleada, difieren en la 
inyección unilateral o bilateral, vía de administración, dosis y lugar anatómico de la inyección.

Sin embargo, la literatura colectiva publicada ha proporcionado una amplia evidencia de la eficacia positiva de la toxina botulinica en el tratamiento de la disfonía espasmódica.

En revisiones sistemáticas se ha evidenciado la eficacia de la toxina botulínica en el tratamiento de la disfonía espasmódica (Whurr, 1998; Boutsen et al., 2002; Faham, 2019).

El estudio de Truong et al. (1991) es el único que comparaba a grupos tratados y no tratados. Cinco son las medidas que recoge cuatro días después de la inyección en ambos grupos. Utiliza la prueba no paramétrica de Mann Whitney U para analizar los resultados significativos de las medidas usadas: análisis espectrográfico, gama de frecuencia fundamental, grado de autopercepción de mejora de la voz y grado espectrográfico profesional. Truong et al. encontraron que la toxina botulinica mejoraba la eficacia básica del funcionamiento fisiológico del mecanismo vocal en cuatro de las cinco medidas relacionadas (frecuencia fundamental, gama de frecuencia fundamental, grado de perturbación, análisis espectrográfico). Además, demostraron que el grado del paciente en la producción del discurso y los grados independientes de la severidad del discurso mejoraron perceptiblemente como resultado del tratamiento. Sin embargo, no se incluye en este estudio los efectos a largo plazo y los efectos secundarios.

Otros autores han publicado que los efectos del tratamiento con toxina botulinica duran entre 3 y 12 meses. Truong et al. (1991) determinaron las medidas postratamiento a los 4 dias y hablaron de que existía una mejora media que duraba unos tres meses. La voz aérea apareció como efecto secundario en dos pacientes. No se proporcionó ninguna información con respecto a la producción de voz más allá de los cuatro dias. Esto está en contraste con otros estudios que divulgan que los efectos positivos de la toxina botulinica no se pueden observar hasta dos semanas después de la inyección de la misma. Sería importante realizar estudios para ver los efectos a más largo tiempo y el grado de severidad de los efectos secundarios. Uno de los problemas que encontramos en los estudios revisados es el tamaño pequeño de las muestras analizadas. Seria conveniente que en los estudios futuros se incluyeran muestras más grandes para aumentar la energía en detectar la presencia de resultados más significativos.

Después de estudiar la bibliografia publicada hasta ahora sobre el tratamiento con toxina botulinica, vamos a describir las diferentes técnicas que existen en el momento actual para la inyección local de la toxina botulinica.

- Infiltración vía transcutánea

Se realiza con una aguja especial, que permite realizar un registro electromiográfico del músculo durante la inyección. El aspecto de la señal eléc- 
trica permite obtener al mismo tiempo la confirmación del diagnóstico y asegurarse de la correcta colocación de la aguja. La dosis de toxina inyectada es muy débil, del orden de 0,5 a $2 \mathrm{mg}$. El efecto de la inyección no es inmediato; se aprecia a las 24-48h con la desaparición de los espasmos vocales, acompañada de una afonía transitoria de varios dias. Puede que aparezcan durante este tiempo alteraciones en la deglución, sobre todo para liquidos. La voz regresa aproximadamente a las dos semanas, dando al paciente de forma progresiva la impresión de una curación completa.

Esta impresión de curación se mantiene durante un tiempo que puede variar entre varias semanas y varios meses según el paciente, tras lo cual los espasmos reaparecen de forma progresiva, incitando al paciente a solicitar una nueva intervención.

Se está observando que el intervalo entre las inyecciones puede aumentar progresivamente con el tiempo, tanto más cuanto el paciente sigue paralelo una reeducación vocal y continúa con sus ejercicios de relajación.

En ciertos casos el paciente desarrolla anticuerpos antitoxina que hacen estas intervenciones sean cada vez menos eficaces.

La disminución de la eficacia se encuentra así mismo en las disfonías espasmódicas llamadas en abducción, en las que se trataría más bien, como ya se ha dicho, de la afectación de la musculatura respiratoria y en particular del diafragma, cuya infiltración no resulta aplicable.

- Infiltración bajo anestesia general vía endoscópica

Paciente en decúbito supino con cuello extendido y mediante laringoscopio rígido y microscopio óptico con aguja rígida curva sobre ambas cuerdas vocales. A los pequeños efectos secundarios que podrian existir tras la inyección añadiriamos los de cualquier anestesia general.

- Bajo anestesia local mediante laringoscopia indirecta

Anestesia tópica con lidocaina, el paciente tira con su mano izquierda de su lengua y bien mediante laringoscopio indirecto o mediante lupa se visualizan ambas cuerdas vocales y con aguja hueca semirrígida y curva se infiltran ambas cuerdas vocales.

- Bajo anestesia local vía nasofibroendoscopia flexible con canal de trabajo

Es la que técnica que más se está imponiendo hoy en día. Realización:

Una media hora antes de realizar la técnica se inyecta vía intramuscular atropina a la dosis de $0,1 \mathrm{mg}$ por cada $10 \mathrm{Kg}$ de peso con el objeto de disminuir la secreción salival. Diazepam 5-10 mg v. oral.

Entre 15-20 minutos antes de la técnica, se realiza anestesia local de ambas fosas nasales con lidocaina impregnada en varias torundas. Es una técnica que requiere dos personas para su realización. 
Preparación previa de la toxina: se introducen $2 \mathrm{ml}$ de suero fisiológico en el bote estándar de toxina botulinica (Botox). Preparamos dos jeringas, una con $0,4 \mathrm{ml}$ de la dilución y otra con 0,1 ml. Purgamos la aguja con la disolución de 0,4 hasta que veamos que sale por la punta, suele ser aproximadamente 0,3, y una vez purgada colocamos la jeringa de 0,1 $\mathrm{ml}$ en la boca de la aguja, que será esta la cantidad administrada en cada cuerda vocal, es decir, 0,1 ml que corresponde a 5 UI de toxina botulinica.

Introducimos el nasofibroscopio e instilamos a través de su canal de trabajo lidovaina en la base de la lengua, epiglotis y cara superficial de ambas cuerdas vocales. Tras comprobar que desaparecen los accesos de tos y que al tocar con la punta de fibroscopio la cara superficial de las cuerdas y no observar reflejo, introducimos aguja (el $2^{\circ}$ cirujano) a través de canal e inyectamos a nivel de $1 / 3$ medio en ambas cuerdas vocales cambiando la jeringuilla en cada cuerda vocal $(0,1 \mathrm{ml}-5 \mathrm{U}$ de toxina- de la dilución en cada cuerda vocal).

Los resultados tras la infiltración de la toxina botulinica son notables y se perciben aproximadamente 20 dias tras la intervención. El efecto de la toxina dura entre 6 y 9 meses, después de lo cual hay que volver a realizar la intervención.

No existen contraindicaciones para el tratamiento y se ha usado con seguridad en la población pediátrica. Hay que tener en cuenta que los aminoglucósidos potencian sus efectos y que los pacientes con diagnóstico de reflujo faringolaringeo deben ser tratados con medicación antirreflujo antes de la intervención. Entre el 3-5\% de los pacientes desarrollan resistencia a la toxina y en estos casos, pueden ser tratados con otros serotipos de la toxina.

\subsection{EVOLUCIÓN SIN TRATAMIENTO ADECUADO}

A falta de suficientes estudios, no es fácil saber cómo evoluciona sin tratamiento adecuado la disfonía espasmódica. Únicamente a través de la anamnesis de los pacientes que acuden a consulta es factible hacerse una idea al respecto.

No obstante, cabe afirmar que la disfonía espasmódica evoluciona lenta y escalonadamente, con ocasión de acontecimientos traumáticos o de terapéuticas inadecuadas, especialmente la recomendación del silencio vocal. No parecen existir regresiones espontáneas, pero se observan estabilizaciones que pueden durar muchos años: ocurre como si el paciente, tras haber luchado contra su trastorno vocal, acabara a veces por acomodarse a él, eso sí, a costa de una cierta disminución de su actividad general.

\subsection{OBJETIVOS}

El protocolo de la European Laringological Society (ELS) para la valoración funcional de la disfonía incluye cinco dimensiones: percepción, análisis 
acústico, videoestroboscopia, aerodinámica y autovaloración del paciente. En este estudio analizamos la efectividad del tratamiento con toxina botulínica en la disfonía espasmódica aductora mediante el análisis acústico de la voz, con el fin de cuantificar la mejoria pre y postratamiento y resaltar la importancia de la utilización de estas herramientas diagnósticas para la objetivación de los resultados.

\section{MATERIALES Y MÉtodoS}

Estudio retrospectivo sobre 113 pacientes diagnosticados de disfonía espasmódica aductora en la Unidad de la Voz del Hospital U. Puerta del Mar desde marzo 2005 hasta enero 2017.

Para el análisis acústico, registramos la voz mediante micrófono conectado a grabadora, con una "a" prolongada pronunciada por el paciente. Se utiliza el programa Praat para el análisis apreciándose, el espectograma, jitter, shimmer, variaciones de la frecuencia fundamental, bloqueos y cociente armónicos ruido antes y después del tratamiento. El tiempo seleccionado para el estudio acústico es de 1,5 segundos de media entre todos los realizados.

Todos los pacientes son tratados con infiltración de botulínica tipo A mediante nasofibrolaringoscopio con canal de trabajo mediante anestesia local con lidocaina al 5\%.

El análisis estadístico de los resultados se realiza mediante el paquete estadístico SPSS versión 21.0. Se realiza la prueba T Student de muestras relacionadas para la significación estadística de la comparación de medias de los patrones acústicos estudiados.

\section{Resultados}

Los pacientes presentan un intervalo de edad entre 18 y 65 años, con una media de $54 \pm 11$.

Hay que destacar que hay una diferencia muy significativa en todos los parámetros acústicos estudiados, comparándolos antes y al mes después de la intervención $(\mathrm{p}<0.001)$, con normalización de los parámetros alterados.

La media de bloqueos antes de la aplicación de la toxina fue de 5,3 $\pm 1,7$, estando ausente en los pacientes a los que se les aplicó la toxina botulínica $(\mathrm{p}<0,001)$

Los resultados del Jitter previo a la intervención se situaban en un rango entre 2,5 y 4 , con una media de $3,22 \pm 0,61$, siendo posterior a la intervención el rango entre 0,4 y 1 con una media de 0,69 $\pm 0,24$ ( $p<0,0001)$.

Los resultados del Shimmer previo a la intervención se situaban en el rango entre 9 y 17, con una media de $13,1 \pm 3,29$, siendo posterior a la intervención el rango entre 3 y 6 , con una media de 4,3 $\pm 1,23(\mathrm{p}<0,0001)$.

El cociente armónicos-ruido en pacientes antes de la aplicación de la toxina oscilaba entre 2,5 y $8 \mathrm{~dB}$, con una media de $5,15 \pm 2,26$, mientras que 
los obtenidos tras la aplicación de la toxina oscilan entre 14-21 dB, con una media de 17,3 $\pm 2,8823(\mathrm{p}<0,0001)$.

Respecto a los efectos adversos, la disfagia y la hipofonía son los más frecuentes, aunque siempre leves y reversibles. La disfagia ha sido referida en un $3 \%$ de los pacientes, durando una media de 6,3 dias, y la hipofonía se produjo en un 38,3\% de los pacientes y duró una media de 8,2 días.

\section{Discusión}

Las medidas de resultados de efectividad utilizadas en los estudios de tratamiento de disfonía espasmódica son muy diversas y variadas.

Nuestro estudio respalda la eficacia del tratamiento de la disfonía espasmódica con toxina botulinica con mediciones objetivas derivadas del análisis acústico de la voz y sugiere que la eficacia de los tratamientos recurrentes es estable a lo largo del tiempo.

La disfonía espasmódica es una enfermedad poco frecuente, poco conocida e infravalorada en muchas ocasiones. Se trata de una patologia con gran impacto en la vida de los pacientes que la padecen, ya que dificulta una función básica, la comunicación.

El diagnóstico de esta patología es fundamentalmente clínico apoyado con las exploraciones instrumentales. Nosotros consideramos fundamental el análisis acústico de todos estos pacientes para poder cuantificar objetivamente el grado de alteración de la voz, así como la magnitud de la mejoría tras el tratamiento.

En la Unidad de Voz del Hospital Universitario Puerta del Mar se realiza una evaluación acústica basándonos en los parámetros de Frecuencia Fundamental (F0), Jitter, Shimmer, bloqueos, cociente armónicos ruido y espectograma. Para el diagnóstico de la disfonía espasmódica también se utilizan estudios aerodinámicos de la voz y la electromiografia laríngea (tiroaritenoidea).

En nuestra serie hemos usado la técnica bajo anestesia local vía nasofibroendoscopia flexible con canal de trabajo, ya que se trata de una técnica sencilla, rápida, bien tolerada por el paciente y segura.

Actualmente, el tratamiento de elección para la disfonia espasmódica aductora es la infiltración intracordal de toxina botulinica, sin embargo, en ningún caso es un tratamiento curativo y los efectos son reversibles en aproximadamente 6 meses.

Los efectos secundarios del tratamiento son leves, poco frecuentes y, en todos los casos de nuestra serie, reversibles.

\section{Conclusiones}

La toxina botulinica es un tratamiento eficaz para la disfonía espasmódica aductora que, además de conseguir una normalización de los parámetros acústicos, es seguro, sencillo de realizar y con pocas complicaciones. 
El análisis acústico de los parámetros vocales nos ofrece una herramienta útil para cuantificar las variaciones en la voz tras el tratamiento, pues permite demostrar objetivamente la mejoría.

\section{REFERENCIAS}

BLITZER A. S. L. et al. (2001): "Botulinum toxin: basic science and clinical uses in otolaryngology", Laryngoscope, 111 , pp. 218-226.

BOONE, Le D. M. S. (2014): The Voice and Voice Therapy, Boston: Boston Pearson.

BOUTSEN, F. et al. (2002): "Botox treatmente for adductor spasmodic dysphonia: a metaanalysis", Journal of Speech, Language and Hearing Research, 45, pp. 469-481.

CANNITO, M. J. P. (1981): "Spastic dysphonia: a continuum disorder", J Commun Disroders, 14 , pp. 215-23.

ESPOSITO, M. et al (2015): "Spasmodic dysphonia followup with videolaryngoscopy and voice spectrography during treatment with botulinum toxin", Neurological Sciences, 36, pp. 1679-1682

FAHAM, M. et al. (2019): "Quality of Life After Botulinum Toxin Injection in Patients With Adductor Spasmodic Dysphonia; a Systematic Review and Meta-analysis", Journal of Voice. Disponible en: https://www.ncbi.nlm.nih.gov /pubmed/31477348 (Fecha de consulta: $10 / 03 / 2020)$.

MURRY, T. W. G. (1995): “Combinedmodality treatment of adductor spasmodic dysphonia with botulinum toxin and voice therapy", Journal of Voice, 9, pp. 460-465.

ROJAS, G. V. E. et al. (2017): "Vocal Parameters and Self-Perception in Individuals With Adductor Spasmodic Dysphonia", Journal of Voice, 31 (3), pp. 391-398.

SINGH S. K. et al. (2016): "A study of voice changes in spasmodic dysphonia after botulinum therapy", Journal of Evolution of Medical and Dental Sciences, 5 pp. 1152-1155.

SPENCER, H. (1908): The Principles of Sociology, Nueva York: Appleton.

STEMPLE, J. C. (2000): Voice therapy: Clinical Studies, St Louis, MO: Mosby Year Book.

TRUONG, D. et al. (1991): "Doubleblind controlled study of botulinum toxin in adductor spasmodic dysphonia", Laryngoscope, 101, pp. 630634.

VOGEL, D. \& CANNITO, M. (2001): "Neurological aspects of spasmodic dysphonia", Treating Disordered Speech Motor Control, Austin: Pro-ed.

WHURR, R. et al. (1993): “The use of botulinum toxin in the treatment of adductor spasmodic dysphonia", Journal of Neurology, Neurosurgery \& Psychiatry, 5, pp. 526-530

WHURR, R. et al. (1998): "Psychological function in spasmodic dysphonia before and after treatment with botulinum toxin", Journal of Medical Speech-Language Pathology, 2, pp. 81-91. 